\title{
Expectations for specialized knowledge in oncology: time to reassess
}

\author{
Ethan Basch
}

Why haven't more medical oncologists embraced complementary and alternative medicine (CAM) therapies, despite the increased patient usage of these therapies? The ESO/EORTC conference proceedings summarized by Lejeune et al., which are supplementary to this article, suggest that usage of CAM therapies in Europe is increasing. Similar trends are reported in the US, with recent surveys finding up to $80 \%$ of American cancer patients using at least one CAM approach since diagnosis. ${ }^{1,2}$ Yet few US oncologists actively discuss CAM therapies with patients, let alone direct the use of these treatments. It's not for lack of effort on the part of policymakers. In the US, the federally funded National Center for Complementary and Alternative Medicine (NCCAM) budgeted over \$122 million for CAM research in 2006. NCCAM's stated mission is to "support rigorous research on CAM, train researchers in CAM, and disseminate information to the public and professionals on which CAM modalities work, which do not, and why."

Have NCCAM's efforts succeeded? From a scientific perspective they have, with a profound improvement in the quality of CAM research overall. Yet findings of NCCAM-funded studies have had limited impact on physician practice patterns or patient usage. For example, highprofile negative studies of saw palmetto, black cohosh, and St John's wort have had little effect on the popularity or sales of these agentsand seem to have just raised questions about design issues in CAM research. Compare this situation to a registration track phase III trial, for which a negative result would essentially end the development of a drug.

This double standard is a cause of confusion to oncologists, who are highly evidence-driven as a profession. For most herbals, definitive evidence is lacking as to what formulation, brand, dose, schedule, or duration is effective and safe. Oncologists generally consider sorting through these complexities to be beyond the boundaries of their expertise, which is probably an appropriate conclusion.
Is there a

context

in which

oncologists

will accept

or even

recommend

CAM

therapies?

E Basch is a Medical Oncologist and Health Services Researcher at Memorial SloanKettering Cancer Center, NY, USA.

Competing interests The author declared he has no competing interests.

www.nature.com/clinicalpractice doi:10.1038/ncponc0815
Is there a context in which oncologists will accept or even recommend CAM therapies? There is ample evidence that the answer is yes - when there is a high level of positive efficacy and safety evidence (or professional consensus), such that a treatment is considered 'standard' rather than 'CAM'. Examples include vitamin B12, folate, or iron for deficiency-based anemia, or calcium and vitamin D for osteoporosis prevention. Pharmaceutical companies devote substantial resources to evaluating natural derivatives, such as paclitaxel, which oncologists would invariably accept following rigorous evaluation and regulatory approval.

The proprietary multi-ingredient herbal formula PC-SPES was embraced by oncologists until its inauspicious end due to tainting. Moreover, the Selenium and Vitamin E Cancer Prevention Trial is often featured at major cancer meetings alongside other chemoprevention trials. Most oncologists are also aware of important potential interactions, such as St John's wort and P450, and the theoretical interference of high-dose antioxidants with some chemotherapeutics or radiotherapy.

It is increasingly recognized that maintaining knowledge beyond this level is not feasible for oncologists, and requires specific expertise. An increasing number of cancer centers are opening integrative medicine divisions, which offer expert CAM consultation services. This multidisciplinary model can also educate oncologists about when referrals are appropriate; for example, to alleviate symptoms or improve quality of life. The needs of patients will be best served in such a collaborative context, in which knowledge expectations (and limitations) of both roles-medical oncologist and CAM consultant-are clearly delineated and accepted.

Supplementary information in the form of a meeting summary and a reference list is available on the Nature Clinical Practice Oncology website. 